\title{
Clinical challenges of a co-secreting TSH/GH pituitary adenoma
}

\author{
Hui Yi Ng', Divya Namboodiri', Diana Learoyd ${ }^{3}$, Andrew Davidson², Bernard Champion ${ }^{1}$ \\ and Veronica Preda ${ }^{1}$ \\ 1Department of Clinical Medicine, Level 4, Macquarie University, 2 Technology Place, Macquarie University, New \\ South Wales, Australia, 2 Department of Neurosurgery, Level 2, Macquarie University, 2 Technology Place Macquarie \\ University, New South Wales, Australia, and 3University of Sydney, Faculty of Medicine and Health, Northern Clinical \\ School, Reserve Road St Leonards, New South Wales, Australia
}

Correspondence should be addressed to V Preda

Email

veronica.preda@mq.edu.au

\section{Summary}

Co-secreting thyrotropin/growth hormone (GH) pituitary adenomas are rare; their clinical presentation and long-term management are challenging. There is also a paucity of long-term data. Due to the cell of origin, these can behave as aggressive tumours. We report a case of a pituitary plurihormonal pit-1-derived macroadenoma, with overt clinical hyperthyroidism and minimal GH excess symptoms. The diagnosis was confirmed by pathology showing elevated thyroid and $\mathrm{GH}$ axes with failure of physiological GH suppression, elevated pituitary glycoprotein hormone alpha subunit (aGSU) and macroadenoma on imaging. Pre-operatively the patient was rendered euthyroid with carbimazole and underwent successful transphenoidal adenomectomy (TSA) with surgical cure. Histopathology displayed an elevated Ki-67 of 5.2\%, necessitating long-term follow-up.

\section{Learning points:}

- Thyrotropinomas are rare and likely under-diagnosed due to under-recognition of secondary hyperthyroidism.

- Thyrotropinomas and other plurihormonal pit-1-derived adenomas are more aggressive adenomas according to WHO guidelines.

- Co-secretion occurs in 30\% of thyrotropinomas, requiring diligent investigation and long-term follow-up of complications.

\section{Background}

Less than $1 \%$ of pituitary adenomas are thyrotropinomas, secreting thyroid-stimulating hormone (TSH), about 30\% co-secrete growth hormone (GH), prolactin (PRL) and $\alpha \mathrm{GSU}$ of thyroid-stimulating hormone (TSH) (1). They are pit-1 staining (2). Plurihormonal, pit-1-positive adenomas and higher Ki-67-staining tumours seem to predict a higher risk of recurrence, compared to mono-secreting tumours. Careful follow-up of these cases is required. Early detection is key to optimise patient management (3).

\section{Case presentation}

A 51-year-old builder presented with an 8-month history of headaches, dizziness, anxiety, palpitations, hypertension, insomnia and heat intolerance. There was no history of visual disturbance nor symptoms to suggest hypothalamic-pituitary axis (HPA) compromise or GH excess. He was initially seen by a cardiologist and otolaryngologist and had cerebral magnetic resonance imaging (MRI), which demonstrated a pituitary macroadenoma. He was referred to a neurosurgeon and then an endocrinologist.

Cardiology assessment demonstrated intermittent sinus tachycardia with mild concentric left ventricular hypertrophy $(11 \mathrm{~mm})$ on transthoracic echocardiogram. Coronary angiogram revealed a 50\% stenosis of left anterior descending artery. He was medically managed with metoprolol $25 \mathrm{mg}$ twice daily (BD), aspirin $100 \mathrm{mg}$ daily and rosuvastatin $5 \mathrm{mg}$ daily. 
Prior to this, there was no other significant medical history. He was a non-smoker and consumed minimal alcohol. Family history included papillary thyroid cancer in his sister, but no multiple endocrine neoplasia (MEN).

On examination, BMI was $29 \mathrm{~kg} / \mathrm{m}^{2}$, blood pressure $157 / 103 \mathrm{mmHg}$, with a regular heart rate of 89 beats per minute. There was a fine peripheral tremor, but no proximal myopathy or orbitopathy. There was a small non-tender nodular goitre. There were no cushingoid or acromegalic features.

\section{Investigations}

Pituitary function revealed TFTs suggestive of secondary hyperthyroidism; normal TSH: $2.37 \mathrm{mIU} / \mathrm{L}(0.4-4)$, raised free T4: $23.1 \mathrm{pmol} / \mathrm{L}(9-19)$ and raised free T3: $8.7 \mathrm{pmol} / \mathrm{L}$ (2.6-6). On review of previous tests, TSH screening had been consistently in the normal range but free $\mathrm{T} 4$ or $\mathrm{T} 3$ were not measured. Thyroid autoantibodies were negative. $\alpha \mathrm{GSU}$ of TSH, sex hormone-binding globulin (SHBG) and calculated $\alpha$ GSU-to-TSH molar ratio (using the $\alpha \mathrm{GSU} / \mathrm{TSH}$ molar ratio $\{[\alpha \mathrm{GSU}(\mu \mathrm{g} / \mathrm{L}) / \mathrm{TSH}(\mathrm{mU} / \mathrm{L})] \times 10\}$ equation (4) were elevated. Insulin-like growth factor 1 (IGF1) was elevated, and GH suppression test demonstrated inadequate $\mathrm{GH}$ suppression of $0.56 \mu \mathrm{g} / \mathrm{L}(<0.4 \mu \mathrm{g} / \mathrm{L})(5)$ (Table 1). Prolactin, morning cortisol, 24-h urinary free cortisol, testosterone and corrected calcium were normal. Thyroid ultrasound showed a multinodular gland and bone mineral density showed vertebral and long bone osteopaenia (Tables 1 and 2).

MRI revealed a homogenously enhancing pituitary lesion measuring $15 \times 12 \times 18 \mathrm{~mm}$ (anteriorposterior $\times$ craniocaudal $\times$ transverse), abutting the undersurface of the optic chiasm (Fig. 1B). Formal visual fields were normal.

\section{Treatment}

He was commenced on carbimazole $15 \mathrm{mg} \mathrm{BD}$ and propranolol $40 \mathrm{mg} \mathrm{BD}$ (metoprolol ceased). At 1 month, he was clinically and biochemically euthyroid; elevated TSH: $12.3 \mathrm{mIU} / \mathrm{L}$, normal free T4: $12.3 \mathrm{pmol} / \mathrm{L}$ and free T3: $4.3 \mathrm{pmol} / \mathrm{L}$. Carbimazole was ceased when he underwent uneventful TSA surgery 3 weeks later. Six weeks post-operatively, TFTs and IGF-1 normalised and there was adequate $\mathrm{GH}$ suppression $(<0.07 \mu \mathrm{g} / \mathrm{L})$ (Table 1). Histopathology revealed a plurihormonal adenoma with strong diffuse nuclear immunoreactivity with PIT-1 antibody. H\&E stains showed a pituitary adenoma composed of a fairly monotonous population of tumour cells with round nuclei, granular chromatin and granular eosinophilic to basophilic cytoplasm. There was TSH, GH, PRL immunopositivity and a raised Ki67 of 5.2\% (Fig. 2A). Immunostains showed positive staining for PIT-1, and TSH (95\%), GH (70\%) and PRL (25\%), with double staining highlighting a population of cells that stain for both hormones with variable combinations (GH/TSH, PRL/GH, and PRL/TSH), in keeping with a plurihomonal PIT-1 adenoma (Fig. 2B and C).

\section{Outcome and follow-up}

Three-month post-operative MRI showed removal of the tumour with post-surgical change (Fig. 1B). He remained clinically and biochemically euthyroid, $\alpha$ GSU of TSH and $\alpha$ GSU-to-TSH molar ratio had normalised. Repeat thyroid ultrasound was unchanged (Table 2). Regular beta-blockade was ceased post-operatively. He will have ongoing surveillance for recurrence.

\section{Discussion}

Thyrotropinomas are rare pituitary adenomas and are classified in the recent WHO guidelines as aggressive tumours that can be locally invasive $(1,3)$. In the last few decades, they have been more frequently reported due to greater clinical awareness, and the advent of $\alpha \mathrm{GSU}$ assays (1). There is no gender or ethnic predilection and patients tend to present at 40-50 years. Twenty-five percent of thyrotropinomas co-secrete other anterior pituitary hormones; most commonly GH (17.9\%), PRL (10.2\%) and gonadotropins (1.8\%) (4). $>75 \%$ of tumours are macroadenomas and are usually associated with increased $\alpha \mathrm{GSU}$, although microadenomas have normal $\alpha \mathrm{GSU}$ levels. Thirty-three percent of invasive macroadenomas occur in patients with prior thyroid ablation due to initial misdiagnosis of central hyperthyroidism as primary hyperthyroidism (1). Our patient fortunately had his pituitary lesion diagnosed first, which led to the diagnosis of central hyperthyroidism. Work-up demonstrated plurihormonality with TSH/T4 discordance, raised GH levels and failure of GH suppression, and discovery of plurihormonal PIT-1 positive adenoma (previously silent type 3) with a high Ki67-5.2\%. As well as the increased proliferation rate being associated with aggressive behaviour in pituitary adenomas, plurihormonal PIT-1 adenomas are known to be aggressive in terms of their size, growth rate, invasiveness and recurrence (3). Conversion to carcinoma or metastatic spread is rare and no causative oncogene has been found (4). 
Table 1 Initial and post-operative biochemical and DEXA results.

\begin{tabular}{|c|c|c|c|c|c|c|c|c|c|}
\hline \multirow[b]{2}{*}{ Parameters } & \multirow{2}{*}{$\begin{array}{l}\text { Initial } \\
\text { bloods }\end{array}$} & \multicolumn{2}{|c|}{ Post-op at } & \multirow{2}{*}{$\begin{array}{c}\text { PG } \\
(3.6-6 \mathrm{mmol} / \mathrm{L})\end{array}$} & \multicolumn{2}{|c|}{ GH } & \multirow{2}{*}{$\begin{array}{c}\text { BMD } \\
(\mathrm{g} / \mathrm{cm})\end{array}$} & \multirow[b]{2}{*}{ T-score } & \multirow[b]{2}{*}{ Z-score } \\
\hline & & 5 days & 6 weeks & & $(0-15 \mathrm{mU} / \mathrm{L})$ & $\mu g / L$ & & & \\
\hline TSH (0.4-4 mlU/L) & 2.37 & 1.13 & 1.84 & & & & & & \\
\hline Free T4 (9-19 pmol/L) & 21.1 & 8 & 15.3 & & & & & & \\
\hline Free T3 (2.6-6 pmol/L) & 8.7 & 2.3 & 3.8 & & & & & & \\
\hline$\alpha G S U(0-0.7$ IU/L) & 2.27 & & 0.48 & & & & & & \\
\hline$\alpha G S U$ to TSH molar ratio & 9.6 & & 2.6 & & & & & & \\
\hline IGF-1 (10-31 nmol/L) & 51 & 30 & 23 & & & & & & \\
\hline Growth hormone (0-15 mIU/L) & 2.8 & 2.9 & 0.2 & & & & & & \\
\hline ACTH (<12.1 pmol/L) & 6 & 4.4 & 5.7 & & & & & & \\
\hline Cortisol (138-650 nmol/L) & 317 & 419 & 428 & & & & & & \\
\hline 24-h urine cortisol (54-319 nmol/day) & 82 & & & & & & & & \\
\hline FSH (1-12 IU/L) & 5.6 & 4.8 & 5.1 & & & & & & \\
\hline LH (0.6-12 IU.L) & 3.2 & 1.5 & 3.1 & & & & & & \\
\hline Testosterone (9.5-28 nmol/L) & 21.9 & 10.4 & 17 & & & & & & \\
\hline SHBG (15-50 mol/L) & 78 & & & & & & & & \\
\hline Prolactin (54-500 mIU/L) & 181 & 95 & 215 & & & & & & \\
\hline TSH receptor antibodies (<1 IU/L) & 0.8 & & & & & & & & \\
\hline Thyroglobulin antibodies (<4.1 IU/ml) & 2.3 & & & & & & & & \\
\hline Thyroid Peroxidase antibody $(<5.6 \mathrm{IU} / \mathrm{ml})$ & $<0.5$ & & & & & & & & \\
\hline \multicolumn{10}{|l|}{ GH Response to $75 \mathrm{~g}$ glucose } \\
\hline \multicolumn{10}{|l|}{ Pre-operatively } \\
\hline Fasting & & & & 4.5 & 3.7 & 1.2 & & & \\
\hline 1 hour & & & & 13.1 & 2.4 & 0.8 & & & \\
\hline 2 hours & & & & 7.1 & 1.7 & 0.6 & & & \\
\hline \multicolumn{10}{|l|}{ Post-operatively } \\
\hline Fasting & & & & 5 & 0.2 & 0.07 & & & \\
\hline 1 hour & & & & 11.1 & $<0.2$ & $<0.07$ & & & \\
\hline 2 hours & & & & 4.6 & $<0.2$ & $<0.07$ & & & \\
\hline \multicolumn{10}{|l|}{ DEXA pre-operatively } \\
\hline L2 to L4 & & & & & & & 1.052 & -1.6 & -1.9 \\
\hline Left femoral neck & & & & & & & 0.813 & -2 & -1.7 \\
\hline Right femoral neck & & & & & & & 0.819 & -1.9 & -1.7 \\
\hline Total left hip & & & & & & & 0.856 & -1.8 & -1.7 \\
\hline Total right hip & & & & & & & 0.844 & -2 & -1.9 \\
\hline
\end{tabular}

ACTH, adrenocorticotropic hormone; $\alpha \mathrm{GSU}$, alpha subunit; BMD, bone mineral density; DEXA, dual-energy X-ray absorptiometry; GH, growth hormone; IGF, insulin growth factor; PG, plasma glucose; SHBG, sex hormone-binding globulin; TSH, thyroid-stimulating hormone.

Thyrotropinomas are reported in the literature to have milder symptoms and signs of thyrotoxicosis than expected, possibly due to the gradual progression of disease (1). Our patient, had more severe cardiac thyroid hormone excess symptoms. This was in part in keeping with progression to a toxic multinodular goitre, due to stimulation of his existing nodular disease. His cardiac symptoms of palpitations and a high resting heart rate of 90, with labile hypertension, were clinically more pronounced, settling post TSA. There is a paucity of data on the cardiac effects of thyrotropinomas in the literature, with only case reports referring to an association of atrial fibrillation and thyrotropinomas (6). Visual field disturbance and headache are present in $20-25 \%$ of thyrotropinomas, due to tumoural mass effect on neurological structures (1). If the thyrotropinoma co-secretes anterior pituitary hormones, patients can have resultant symptoms of GH, PRL or gonadotrophin excess, such as tissue overgrowth, galactorrhoea or amenorrhoea. These symptoms have been reported to

Table 2 Thyroid ultrasound pre and post-operatively. 

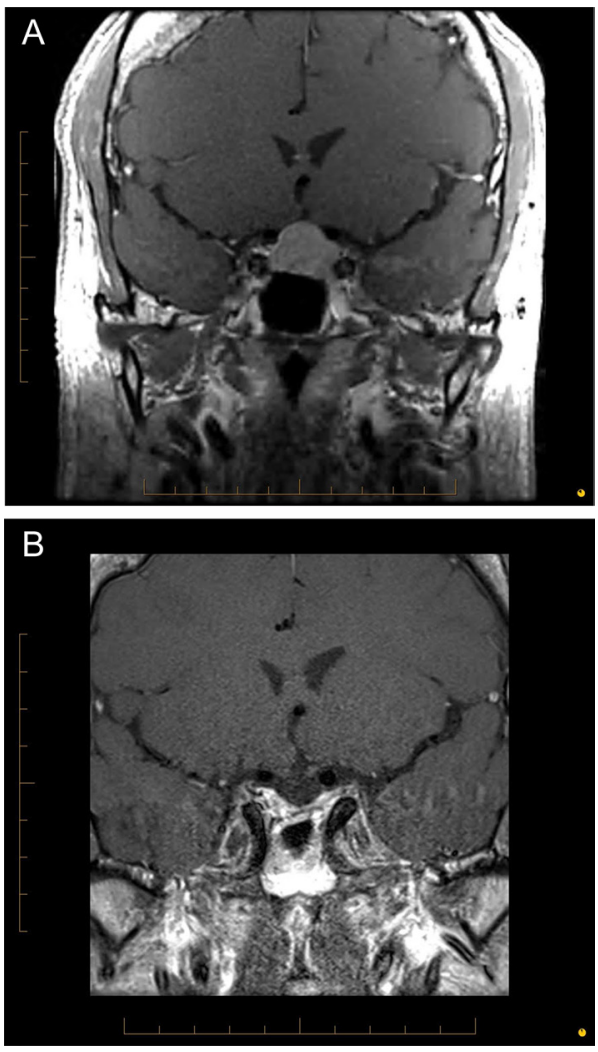

Figure 1

Pre and post-operative MRI images. (A) Large homogenously enhancing lesion $15 \times 12 \times 18 \mathrm{~mm}$ (AP $\times$ craniocaudal $\times$ transverse), abutment of the undersurface of the optic chiasm. (B) 3-month post-op: removal of pituitary macroadenoma, residual pituitary gland posteriorly.

overshadow symptoms of hyperthyroidism (7), but were not evident in our patient. The gonadal axis is frequently affected, presenting as menstrual irregularity in women and decreased libido or central hypogonadism in men, the latter present in our patient (8).

$\alpha \mathrm{GSU}$ or calculated $\alpha \mathrm{GSU}$-to-TSH ratio is elevated (except in microadenoma) due to increased secretion of $\alpha G S U$ by thyrotropinomas. This can also occur in other pituitary adenomas but is not reliable in post-menopausal women; the ratio can be high as 29.1 , compared to 5.7 in normal people (7). Markers of peripheral thyroid activity including SHBG and C-terminal telopeptide (CTX) can be elevated, except in GH co-secretion, which inhibits SHBG (8). Our patient's SHBG was elevated at $78 \mathrm{nmol} / \mathrm{L}$, possibly because there was mild GH excess.

Dynamic testing may be considered when the diagnosis is in doubt. Thyroid-releasing hormone (TRH) stimulation results in a four-fold rise in TSH in normal patients, but in thyrotropinomas, there is a blunted response. T3 suppression (contraindicated in elderly patients and coronary artery disease) should lead to TSH
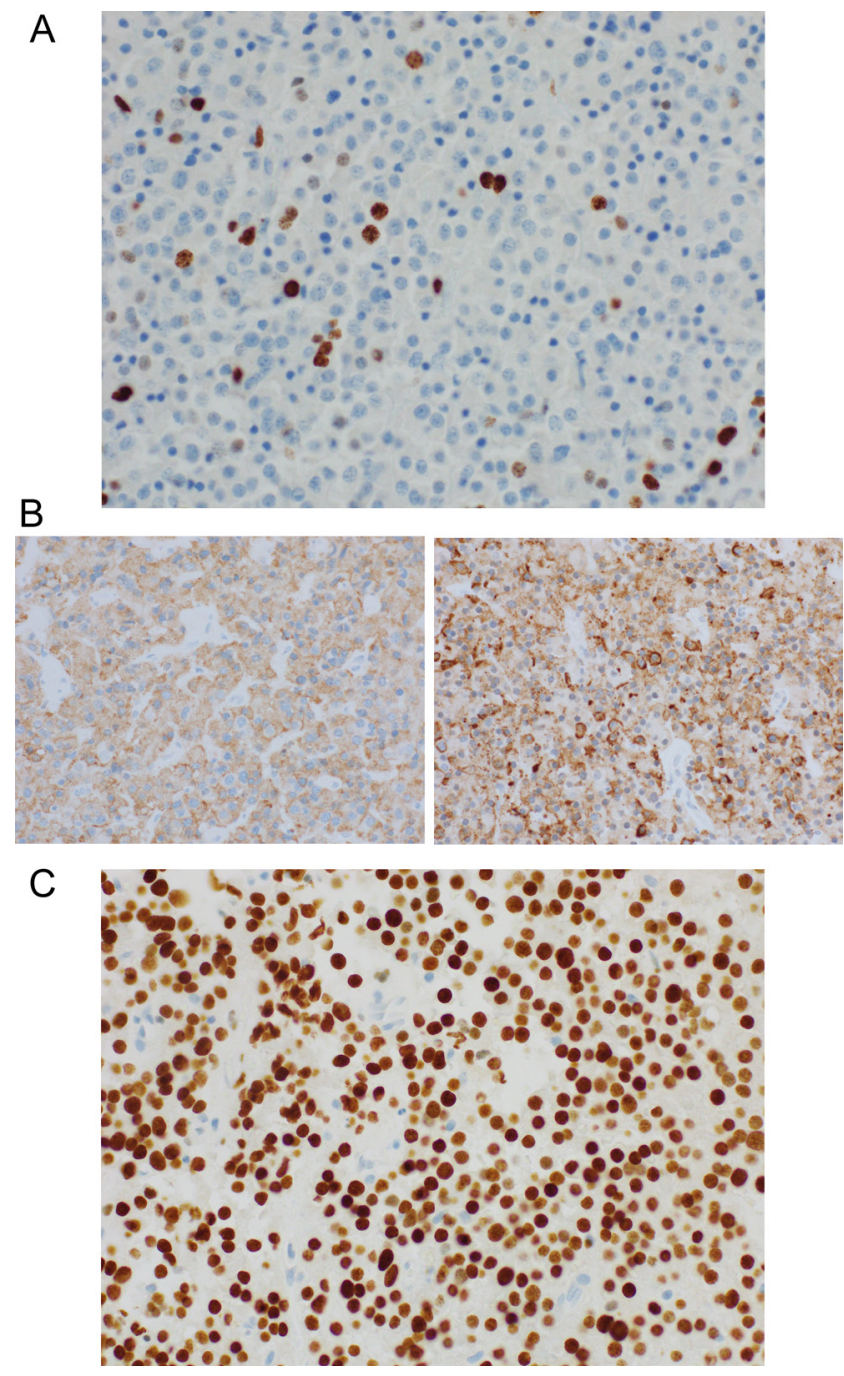

Figure 2

Histopathology. (A) Ki67 staining $\times 40$. Six mitotic figures were identified per HPF. Ki-67 showed increased proliferation (5.2\%). (B) TSH and GH stains $\times 40$ power. Immunostains showed positive staining for PIT- 1 and positive staining for TSH ( $95 \%$ of tumour cells), GH (70\%), in keeping with a plurihomonal PIT-1 adenoma. (C) Pit-1 staining at high power $\times 40$.

suppression in normal patients, but failure to suppress in thyrotropinoma (9). Complete TSH suppression from T3 has never been reported in a thyrotropinoma and is one of the more robust criteria that indicate cure post therapy $(4,7)$. Another discriminatory test is a minimum 2-month administration of somatostatin analogues (SSAs); resultant fall in free T3 and free T4 is strongly indicative of thyrotropinoma (4).

The main differential diagnosis for thyrotropinomas is thyroid hormone resistance (THR), due to the similar biochemical picture of raised or inappropriately normal TSH with raised free $\mathrm{T} 4 \pm$ free $\mathrm{T} 3$. This is provided assay interference including heterophile antibodies and 
Table 3 Factors favouring thyrotropinomas vs thyroid hormone resistance $(2,4,9)$.

Neurological compressive symptoms
Anterior-posterior hormonal c-secretion
Raised $\alpha$ GSU, SHBG, CTx
Family history
TRH stimulation
T3 suppression
Response to SSA
Pituitary adenoma

\begin{tabular}{l}
\hline Favouring thyrotropinoma \\
\hline Present \\
Present \\
Present \\
None except MEN1 \\
Blunted response of TSH \\
Failure to suppress TSH $(<40 \%)(8)$ \\
Present \\
Present
\end{tabular}

Favouring thyroid hormone resistance

Absent

Absent

Absent

Present

Normal to exaggerated response of TSH

Partial dose dependent suppression (>80\%) (8) Absent

Absent

$\alpha$ GSU, pituitary glycoprotein alpha subunit; CTx, C-terminal telopeptide; MEN1, multiple endocrine neoplasia1; SHBG, sex hormone-binding globulin; SSA, somatostatin analogue; TRH, thyroid-releasing hormone; TSH, thyroid-stimulating hormone.

familial dysalbuminaemic hypothyroxinaemia have been excluded (Table 3).

The gold standard of therapy is pituitary surgery to remove the adenoma. This may be incomplete due to high tumour volume, invasion into local structures and marked fibrosis. Patients should be rendered euthyroid pre-operatively through anti-thyroid medications, propranolol or SSA. The pre-operative medical therapy may however affect the post-operative thyroid biochemical findings, making it difficult to determine if patient has been cured (1). Our patient was prescribed carbimazole pre-operatively and was euthyroid prior to surgery. Carbminazole was chosen because of ease of access, patient preference for oral therapy and definitive surgery performed in a timely manner.

Surgery restores euthyroidism in $75-85 \%$ of patients (8); the remainder require adjunctive medical therapy, which our patient has not needed to date. Thyrotropinomas have dopamine receptors and thus dopamine agonists such as cabergoline and bromocriptine have been trialled with variable results; more success has been reported in PRL co-secreting thyrotropinomas. SSAs are far more effective; they reduce TSH and aSU secretion and restore euthyroidism in almost all cases, reduce pituitary mass in 50\%, improve visual impairment in the majority of cases and reduce goitre size in $1 / 5$ th of patients $(1,10)$. SSAs are now the medical mainstay of therapy for thyrotropinomas. Interestingly, the highest somatostatinbinding site densities are in mixed $\mathrm{GH} /$ thyrotropinomas, rendering SSA a good option for our patient if required $(1,10)$. Resistance to SSA is rare and overall tolerance is good; gastrointestinal side effects are generally transient (8). Over-therapeutic TSH suppression from SSA therapy and consequent biochemical hypothyroidism may occur and a block and replace (L-T4) therapy may be needed (4).

Patients who are not cured despite the above measures or in whom surgical/medical therapy is contraindicated, may require fractionated stereotactic radiotherapy, normalising thyroid function in $25 \%$ of patients within 2 years $(8)$.

There are no universally accepted criteria for cure. Clinical and biochemical reversal of thyroid excess is the desired outcome post intervention; however, this can occur when there has been incomplete resection of the tumour. Nevertheless an undetectable TSH, 1 week post-operatively is a good prognosis (1). Our patient's detectable TSH could have been stimulated by low free T4 and free T3 secondary to carbimazole, which continued up until the day of surgery. Other indicators of cure include normalisation of previously raised $\alpha \mathrm{GSU}$ and $\alpha \mathrm{GSU}$-toTSH molar ratio and resolution of other anterior pituitary hormone co-secretion. Our patient's post-operative $\alpha \mathrm{GSU}$ was $0.48 \mathrm{IU} / \mathrm{L}$ and his $\mathrm{GH}$ suppression test was consistent with cure of acromegaly (5). In addition, normalisation in previously abnormal TRH and T3 dynamic testing indicates complete tumour removal; especially, the latter since there has never been complete inhibition of T3 documented in thyrotropinomas (7).

There is a paucity of data on long-term outcomes, but it appears that recurrence of adenoma is uncommon, especially in the first few years post-operatively. Longer studies are needed and patients require ongoing clinical biochemical and radiological follow-up (4).

Thyrotropinomas are rare, more aggressive tumours that can be difficult to diagnose and treat and may be plurihormonal. Our patient's clinical presentation had predominant cardiovascular symptoms, despite a co-secreting tumour. These symptoms improved postoperatively with weaning of beta-blockers. First-line therapy for such tumours is surgery, which may not result in complete cure due to the fibrotic nature and invasiveness of the tumour. SSA is the most effective adjunctive medical therapy that is well tolerated. Long-term follow-up is required for recurrence and complications of thyroid excess. 


\section{Declaration of interest}

The authors declare that there is no conflict of interest that could be perceived as prejudicing the impartiality of the research reported.

\section{Funding}

This research did not receive any specific grant from the funding agencies in the public, commercial or not-for-profit sectors.

\section{Patient consent}

Written informed consent was obtained from the patient for publication of the submitted article and accompanying images.

\section{Author contribution statement}

Divya Namboodiri, Diana Learoyd, Andrew Davidson, Bernard Champion, Veronica Preda - contribution to manuscript writing and revision. Permission obtained from primary physician Bernard Champion for case report submission.

\section{Acknowledgements}

The authors would like to acknowledge and thank Dr Yasmin Matthews, BMed, PhD, FRCPA from Douglas Hanly Moir Pathology and Dr Sophie Corbett-Burns MB, BCh, BAO, FRCPA, Douglas Hanly Moir Pathology.

\section{References}

1 Beck-Peccoz P \& Persani L. Thyrotropinomas. Endocrinology and Metabolism Clinics of North America 200837 123-134, viii. (https:// doi.org/10.1016/j.ecl.2007.10.001)
2 Lee JC, Pekmezci M, Lavezo JL, Vogel H, Katznelson L, Fraenkel M, Harsh G, Dulai M, Perry A \& Tihan T. Utility of Pit-1 immunostaining in distinguishing pituitary adenomas of primitive differentiation from null cell adenomas. Endocrine Pathology 201728 287-292. (https://doi.org/10.1007/s12022-017-9503-6)

3 Nishioka H \& Inoshita N. New WHO classification of pituitary adenomas (4th edition): assessment of pituitary transcription factors and the prognostic histological factors. Brain Tumor Pathology 2018 35 57-61. (https://doi.org/10.1007/s10014-017-0307-7)

4 Beck-Peccoz P, Lania A, Beckers A, Chatterjee K \& Wemeau JL. 2013 European Thyroid Association guidelines for the diagnosis and treatment of thyrotropin-secreting pituitary tumors. European Thyroid Journal 20132 76-82. (https://doi.org/10.1159/000351007)

5 Cazabat L, Souberbielle JC \& Chanson P. Dynamic tests for the diagnosis and assessment of treatment efficacy in acromegaly. Pituitary 200811 129-139. (https://doi.org/10.1007/s11102-008-0113-7)

6 Li J, Tan H, Huang J, Luo D, Tang Y, Yu R \& Huang H. Case report of recurrent atrial fibrillation induced by thyrotropin-secreting pituitary adenoma with Graves' disease. Medicine 201897 e11047. (https:// doi.org/10.1097/MD.0000000000011047)

7 Losa M, Giovanelli M, Persani L, Mortini P, Faglia G \& Beck-Peccoz P. Criteria of cure and follow-up of central hyperthyroidism due to thyrotropin-secreting pituitary adenomas. Journal of Clinical Endocrinology and Metabolism 199681 3084-3090. (https://doi. org/10.1210/jcem.81.8.8768879)

8 Beck-Peccoz P, Persani L \& Lania A. Thyrotropin-Secreting Pituitary Adenomas: Endotext, 2019. (available at: https://www.ncbi.nlm.nih. gov/books/NBK278978/)

9 Chiang C, Inder W, Grossmann M, Clifton-Bligh R, Coates P, Lim EM, Ward P, Standford P, Florkowski C \& Doery J. Harmonisation of Endocrine Dynamic Testing (Adults): Australasian Association of Clinical Biochemists, 2019. (available at: https://www.aacb.asn.au/ aboutus/harmonisation-of-endocrine-dynamic-tests/harmonisationof-endocrine-dynamic-testing-adults)

10 Kuhn JM, Arlot S, Lefebvre H, Caron P, Cortet-Rudelli C, Archambaud F, Chanson P, Tabarin A, Goth MI, Blumberg J, et al. Evaluation of the treatment of thyrotropin-secreting pituitary adenomas with a slow release formulation of the somatostatin analog lanreotide. Journal of Clinical Endocrinology and Metabolism 200085 1487-1491. (https://doi.org/10.1210/jcem.85.4.6548)

Received in final form 8 July 2019

Accepted 16 July 2019 